\title{
La productividad científica en revistas arbitradas de los investigadores del Instituto de Investigaciones en Matemáticas Aplicadas y en Sistemas
}

\author{
Scientific productivity of the Instituto de Investigaciones en Matemáticas Aplicadas
}

$y$ en Sistemas researchers in referred journals

\section{Edgar Martín Barrientos Cruz y Suyin Ortega Cuevas}

Instituto de Investigaciones en Matemáticas Aplicadas y en Sistemas. Universidad Nacional Autónoma de México. México, D.F. C.P. 04510. edgar@leibniz.iimas.unam.mx, suyin@leibniz.iimas.unam.mx.

\begin{abstract}
Resumen
Se analiza la productividad científica de los investigadores del Instituto de Investigaciones en Matemáticas Aplicadas y en Sistemas (Mexico), mostrando un panorama de dónde se publica, en que tipo de revistas, así como en qué disciplinas está presente el Instituto, según la clasificación del ISI (Internacional Scientific Institute).
\end{abstract}

Palabras clave: Revistas arbitradas. Matemáticas. Indización. Factor de Impacto.

\section{Introducción}

El número de artículos publicados en revistas científicas es un indicador de la productividad de la investigación científica que se realiza en un instituto o centro, por lo que es importante saber dónde publican los investigadores $y$, por supuesto, en que tipo de documentos lo realizan.

El Instituto de Investigaciones en Matemáticas Aplicadas y en Sistemas (IIMAS) es un instituto que se enfoca al estudio de las matemáticas aplicadas en todos los campos del conocimiento y, por supuesto, destaca la aplicación de los sistemas, lo que le da un enfoque interdisciplinar. Por ende, los documentos que se generan se envían a diversas revistas de todo el mundo, tomando en cuenta primeramente la especialidad, así como el prestigio de la revista.

Lo anterior hace surgir las siguientes inquietudes: ¿dónde publican los investigadores del IIMAS? ¿en qué tipo de revistas? y ¿en qué lugares?, entre otras interrogantes. Todas ellas ponen de manifiesto la necesidad de realizar un análisis bibliométrico del comportamiento de la productividad científica en el IIMAS.

\begin{abstract}
The scientific productivity of the Instituto de Investigaciones en Matemáticas Aplicadas y en Sistemas (Mexico) researchers is analysed, showing a panorama of the countries and journals where their papers were published, as well as the different disciplines in which this Institute is involved, according to the ISI classification.
\end{abstract}

Keywords: Arbitrated journals. Mathematics. Indexing. Impact Factor.

El presente estudio analiza la información generada durante el periodo de 1997 a 2006 . Estos 10 años se consideraron representativos puesto que desde 1997 se tiene un control exacto de los artículos reportados en los informes de actividades del Instituto y la calidad del control bibliográfico es del $100 \%$.

Se realizan varios análisis de los datos obtenidos. Entre ellos es importante mencionar que se consultó el factor de impacto (número de artículos entre el número de citas de una determinada revista) de este período, que publica el Institute Scientific Information (ISI).

Este pequeño panorama de la productividad científica de los investigadores del IIMAS da una idea de la importancia de la investigación en el área de las matemáticas aplicadas en la mayoría de las disciplinas del conocimiento, remarcando la calidad de la investigación que se realiza en este Instituto. 


\section{La Universidad Nacional Autónoma de México}

La Universidad Nacional Autónoma México (UNAM) ocupa el lugar 74 según la clasificación mundial de universidades que se publicó en el periódico The Times (2006), colocándola como la mejor universidad de Iberoamérica (Boletín UNAM-DGCS-740). Esta información es a nivel mundial, puesto que la evaluación se realizo a todas las universidades existentes.

Por su parte, en México, el Consejo Nacional de Ciencia y Tecnología (CONACyT), a través del Informe General del Estado de la Ciencia y la Tecnología, dentro de la información que proporciona, presenta las diez instituciones que más artículos realizaron en el periodo de 20002005 , donde la UNAM ocupa el primer lugar con 14.528 artículos, lo que equivale al $47,02 \%$ a nivel nacional.

Bajo esté marco, la investigación que se realiza en la UNAM se organiza en dos coordinaciones: "de Humanidades" y "de la Investigación Científica". La Coordinación de Humanidades cuenta con 5 centros de investigación, un Centro de Enseñanza de Lenguas Extranjeras y 10 institutos en humanidades. La Coordinación de la Investigación Científica cuenta con 19 institutos y 9 centros, así como la Dirección General de Divulgación de la Ciencia. El Instituto de Investigaciones en Matemáticas Aplicadas y en Sistemas (IIMAS) es uno de los 19 institutos de la Coordinación de la Investigación Científica.

\subsection{El Instituto de Investigaciones} en Matemáticas Aplicadas y en Sistemas

Oficialmente, el IIMAS surge el 10 de marzo de 1976, y a partir de ahí ha contado con personal académico y administrativo para la realización de sus funciones.

El objetivo principal del IIMAS, es "realizar investigación científica en matemáticas aplicadas, en sistemas y en ciencia e ingeniería de la computación; a parte de participar en la docencia en los programas de posgrado de donde el Instituto es la sede".

\subsubsection{Estructura}

El IIMAS esta organizado de la siguiente manera. En el apoyo académico, se cuenta con un Consejo Interno, dos comisiones (la Dictaminadora y la Evaluadora), y tres secretarías (académica, técnica y administrativa). En la investigación, está organizado en dos áreas: "Matemáticas Aplicadas y Sistemas" y "Ciencia e Ingeniería de la Computación". La primera tiene asociados a cuatro departamentos -Matemáticas y Mecánica (MyM), Métodos Matemáticos y Numéricos (MMyN), y Modelación Matemática de Sistemas Sociales (MMSS)—; y la segunda a dos -Ciencias de la Computación (CC), e Ingeniería de Sistemas Computacionales y Automatización (ISCA).

De acuerdo con el último Informe de Actividades (2006) del IIMAS, la descripción de la labor de los departamentos es la siguiente:

Matemáticas y Mecánica. Está integrado por investigadores cuyo interés principal está enfocado en las matemáticas aplicadas. El lenguaje natural y común del departamento es el de las ecuaciones diferenciales. Un aspecto esencial de las matemáticas aplicadas que cultiva el departamento es la interacción que tienen con muy diversos campos de la ciencia, que permite establecer una comunicación natural y proporcionar marcos conceptuales que representan fenómenos de origen físico, químico, biológico, etcétera...

Métodos Matemáticos y Numéricos. La función primordial consiste en realizar investigación sobre análisis matemático, análisis combinatorio y métodos geométricos en mecánica, con énfasis en la generación de nuevos métodos para la solución de problemas de la física, la química y las ingenierías, así como en el estudio de aspectos matemáticos de métodos existentes...

Modelación Matemática de Sistemas Sociales. Este departamento se dedica al estudio de procesos sociales desde la perspectiva de los sistemas sociales y las redes, así como al estudio de la historia de la ciencia...

Probabilidad y Estadística. La investigación que se realiza en este departamento cubre diferentes áreas de la estadística. Se hace investigación metodológica básica, pero también se desarrollan modelos para descubrir fenómenos de otras disciplinas. Parte de esta investigación tiene que ver con aplicaciones concretas a problemas de relevancia nacional, a saber: modelación de epidemias, modelos sobre contaminación, medio ambiente y otros fenómenos ecológicos, conteos rápidos, construcción de tablas de mortalidad, evaluación de opciones financieras y diseños óptimos de experimentos...

Ciencias de la Computación. El departamento tiene como finalidad realizar investigación básica y aplicada en ciencias de la computación, apoyar y fortalecer el Posgrado en Ciencia e Ingeniería de la Computación, así como a los diversos programas de licenciatura de la UNAM relacionados con esta disciplina científica, además de promover y difundir a las ciencias de la computación..." (Informe de actividades 2006).

Ingeniería de Sistemas Computacionales y Automatización. Que cuenta con dos secciones, a saber:

Sección de Ingeniería de Sistemas Computacionales. Tiene como misión realizar investigación, tanto básica como aplicada, en ingeniería de sistemas computacionales de alto desempeño y confiabilidad, 
formar especialistas de alto nivel en las diversas áreas asociadas a estas actividades de investigación, así como difundir y aplicar los resultados obtenidos para favorecer el avance científico y tecnológico de nuestro país...

Sección de Electrónica y Automatización. Se estudian y desarrollan tecnologías en las áreas de electrónica y automatización para su aprovechamiento con la aplicación a diferentes áreas de investigación. Entre sus objetivos están el estudio y uso de las tecnologías emergentes en el diseño de sistemas digitales para aplicaciones como instrumentación, automatización, comunicaciones y procesamiento de señales e imágenes....

Las líneas de investigación que realizan los departamentos son 52. Además, se desarrollan proyectos internos y externos al IIMAS, en donde participa el personal académico. En algunos de ellos, se colabora con diversas instituciones tanto mexicanas como extranjeras. El personal académico del IIMAS estaba integrado a 31 de diciembre de 2006 por 52 investigadores y 39 técnicos académicos. El nivel de estudios se muestra en la Tabla I.

\begin{tabular}{lrr}
\hline $\begin{array}{l}\text { Grado } \\
\text { de estudios }\end{array}$ & Investigadores & $\begin{array}{c}\text { Técnicos } \\
\text { Académicos }\end{array}$ \\
\hline Doctorado & 48 & 1 \\
\hline Maestría & 1 & 3 \\
\hline Licenciatura & 3 & 39 \\
\hline Total & 52 & 13 \\
\hline
\end{tabular}

Tabla I. Grado de estudios

\section{Metodología}

A partir del año de 1997, la Secretaría Académica creo una base de datos en donde se concentran los artículos que realizan los investigadores del IIMAS. Cada uno de ellos es entregado con los datos de la ficha bibliográfica correspondiente. A través de los años, esta base de datos se ha ido actualizando concentrando toda la información. Se cuenta con el apoyo de la Biblioteca para la localización física de los artículos o los datos bibliográficos que no se tienen con una certidumbre adecuada.

La base de datos cuenta actualmente con 539 registros bibliográficos, los campos que consideran son: autor, coautores, titulo, país, año de publicación, Internacional Standard Serial Number (ISSN), factor de impacto (F.I.), áreas temáticas a las que pertenece según el International Scientific Information (ISI) y su posición. Como herramienta adicional se consultó el directorio de publicaciones periódicas Ulrich's para completar los registros que hicieran falta o bien, para verificar el título correcto, en caso de duda.
Para obtener el F.I de las revistas, se consultó el Journal Citation Report a través del portal Web of Science, editado por el ISI. La Universidad actualmente suscribe en línea los años 2000 a 2006. Para los años retrospectivos de 1997 a 1999, se acudió a la Subdirección de Servicios de Información Especializada de la Dirección General de Bibliotecas de la UNAM para consultarlos en disco compacto.

El método de análisis estadístico, se basa en un estudio retrospectivo, descriptivo, observacional y transversal de la información, en el que se emplea la estadística descriptiva como herramienta para poder visualizar de una manera clara las características de la producción del IIMAS.

Es importante señalar que para ello, se consideró la existencia de dos artículos que fueron publicados en coautoría por investigadores adscritos a distintos departamentos del IIMAS, que fueron contados de manera doble para fines estadísticos al construir gráficas por departamento y, como un sólo registro para la construcción de gráficas globales.

Se emplean líneas de tendencia tanto lineal como por promedios móviles (con periodos de 2 años), para observar si la producción tiende a crecer o disminuir, de manera general.

El método de promedios móviles tiene como objetivo eliminar de una serie de datos las componentes estacionales o accidentales, es decir, suaviza una curva cuando existen muchos cambios bruscos o movimientos irregulares. El método consiste en obtener los promedios de la suma de cada dato con los datos de los "n" años anteriores, de acuerdo al número de años a promediar que se haya establecido previamente. Para el caso de este análisis, se consideraron promedios de dos años. Este método se aplica para presentar la producción de artículos publicados en revistas arbitradas, eliminando los datos extremos (altos y bajos) del periodo que se analiza, permitiendo mostrar más claramente la tendencia de la producción.

Respecto a las revistas en las que aparecen los artículos publicados, se identificaron los factores de impacto para los años de publicación, así como todas las áreas temáticas relacionadas y sus posiciones en las mismas, a través del ISI.

Se obtuvo un total de 434 registros temáticos, los cuales fueron agrupados hasta donde se consideró conveniente, a fin de que reflejaran generalidad, sin perder diversidad. Cabe mencionar que para la traducción de las áreas temáticas del ISI, se consultaron listas de encabezamientos de materia bilingüe. 
Asimismo, se construyeron histogramas de frecuencias para poder determinar en que tipo de revistas se está publicando y qué tan relevantes son, haciendo uso del factor de impacto y de las posiciones en las áreas temáticas.

Para la construcción de los histogramas de frecuencias, se consideró adecuado presentar cinco intervalos para poder contar con una visualización de los datos semejante a una categorización cualitativa de: bajo, medio-bajo, medio, medio-alto y alto.

Además, con el fin de poder contar con una gráfica general de revistas que muestre la posición que ocupan dentro de las áreas temáticas donde se han publicado artículos, se construyó un histograma. Para ello, se consideró conveniente tomar el valor numérico de las posiciones $p=x / y$ que tiene cada revista en las áreas que aparece, donde $x$ representa la posición de la revista dentro del total de las $y$ revistas que integran cierto área.

Es importante hacer notar que el valor $p$, siempre estará en el intervalo $0<p \leq 1$, por lo que, si se multiplica por 100 , puede verse como un valor porcentual que alcanza como máximo valor el $100 \%$ en los casos en que una revista ocupa el último lugar en cierta área, y casi cero en los casos en que la revista se encuentre en la primera posición. Por lo que, para ser congruentes con la interpretación, se consideró más conveniente tomar el valor porcentual complementario de $p$, al que se le denominó $q$, definido como $q=(1-p) 100$, donde los valores grandes de $q$ son de aquellas revistas mejor posicionadas, y los valores pequeños serán para aquellas menos posicionadas. Con ello, se forma un histograma que ayuda a representar el número de revistas que se encuentran en una posición tal que superan el valor " $q$ " por ciento de las revistas que integran cierta área temática.

Por otra parte, para saber en qué índices aparecen las revistas en las que se publica, se procedió a identificarlos a través del directorio de publicaciones periódicas Ulrich's, considerando los 539 registros de artículos.

\section{Análisis Estadístico}

Durante el periodo de 1997-2006, el IIMAS ha producido un total de 539 artículos en revistas científicas arbitradas como producto de la investigación que se desarrolla en las áreas que se cultivan en el Instituto.

Del análisis realizado y tomando como referencia los artículos publicados (Figura 1), se presenta la distribución de dicha producción a lo largo del periodo de 10 años, tanto por depar- tamentos como de manera global. Puede observarse que en las líneas de tendencia, tanto por regresión lineal como por el método de promedios móviles (para un periodo de dos años), muestra una tendencia ascendente.

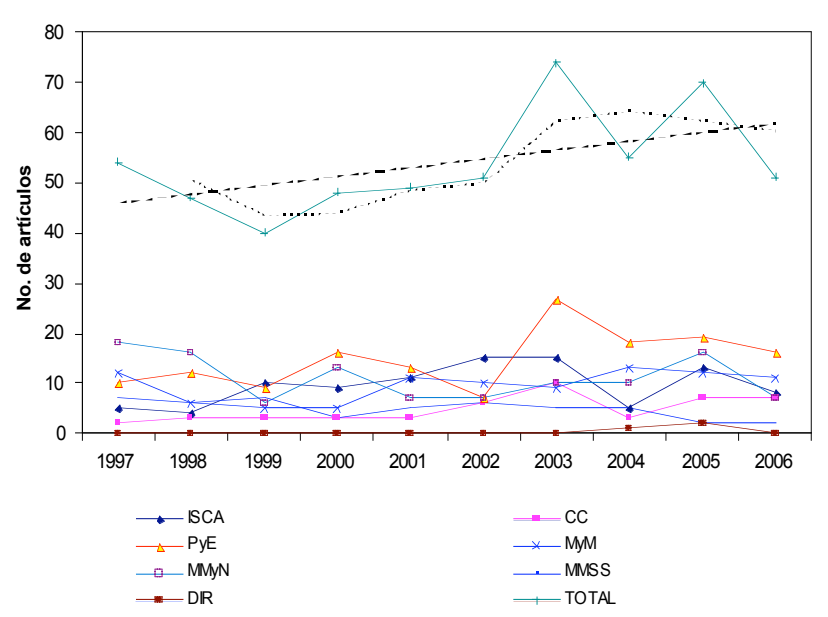

Figura 1. Artículos publicados en revistas, por departamento (1997-2006).

Del total de artículos, el $89 \%$ (479) se publicó en revistas indexadas y el $11 \%(60)$ en revistas que no aparecen en ningún índice (Figura 2).

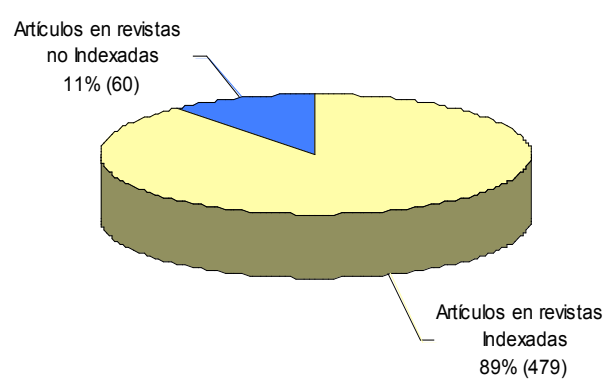

Figura 2. Total de artículos publicados por tipo de revista (1997-2006).

Cabe señalar, que durante todos los años que integran el periodo, la producción de artículos en revistas se realizó principalmente en revistas indexadas, tal como se muestra en la Figura 3.

En lo que respecta a los artículos publicados en revistas indexadas, el $34,7 \%$ (166) se publicó en revistas de Estados Unidos, el 19\% (91) en revistas del Reino Unido, el $14,4 \%$ (69) de Holanda, el $12,5 \%$ (60) de México, el 3,1\% (15) de Alemania y el $1,7 \%$ (8) de España, principalmente, tal como se muestra en la Figura 4. 


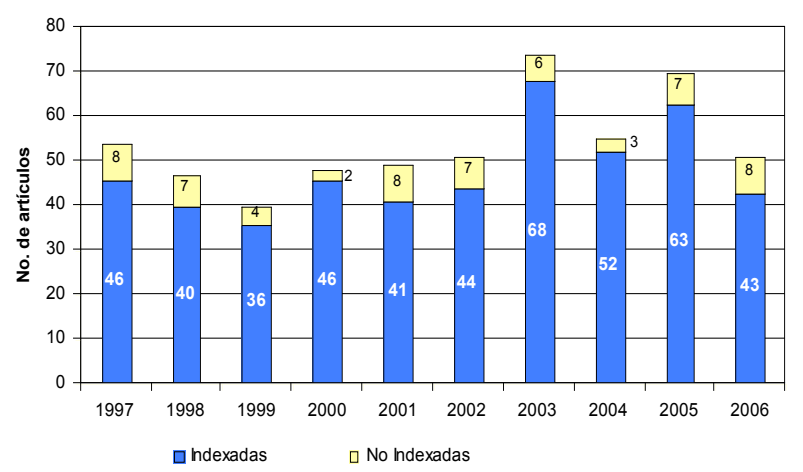

Figura 3. Distribución total de artículos en revistas, por tipo de indexación (1997-2006).

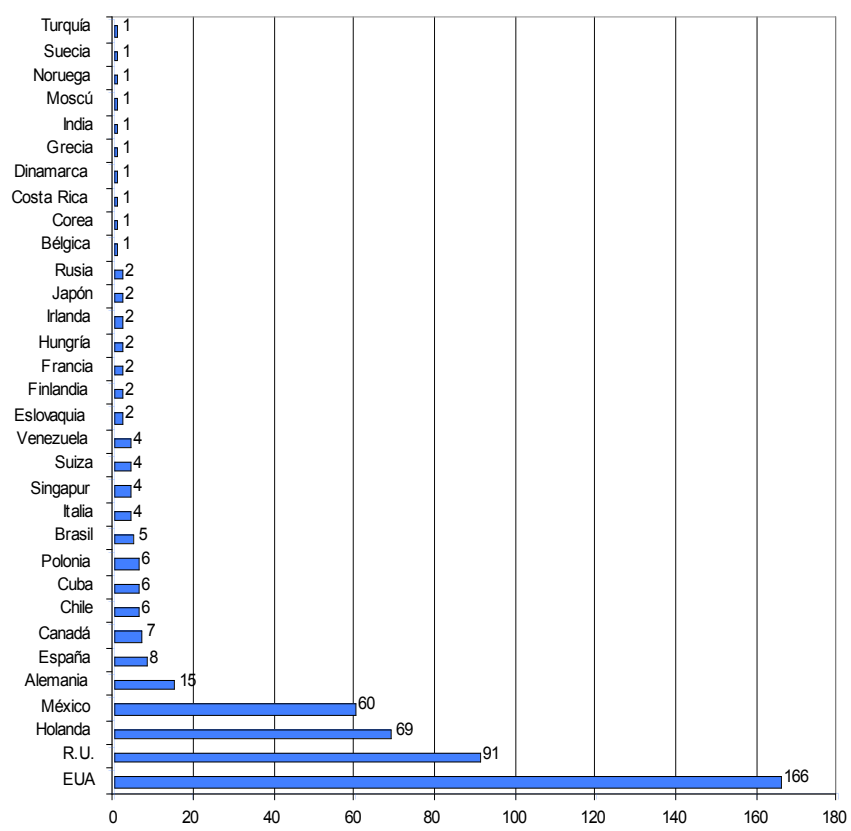

Figura 4. Distribución de artículos en revistas indexadas, por país (1997-2006).

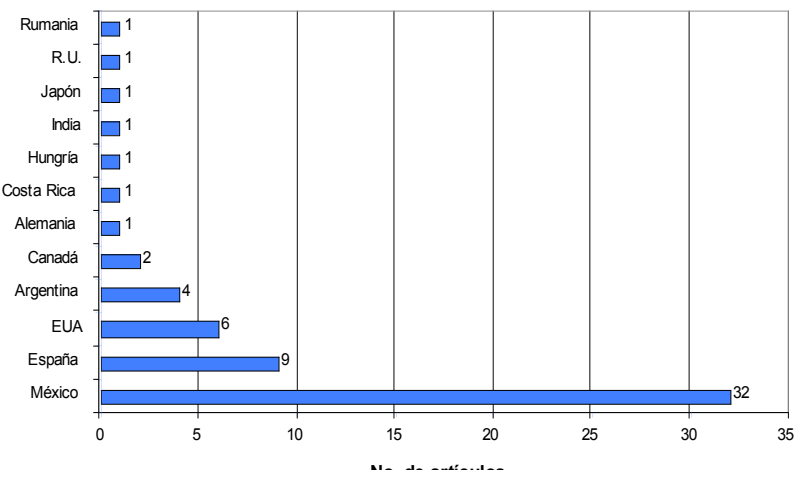

Figura 5. Distribución de artículos en revistas no indexadas, por país (1997-2006).
Por su parte, de los artículos publicados en revistas no indexadas, el 53,3\% (32) se publicó en revistas de México, el 15\% (9) de España, el $10 \%$ (6) de Estados Unidos y el 6\% (4) de Argentina, principalmente (Figura 5).

Ahora bien, tomando como referencia las revistas en las que se ha publicado, la producción del IIMAS está presente en 322 distintas revistas, donde el $87 \%$ de ellas está indexada y el $13 \%$ no aparece en ningún índice de revistas (Figura 6).

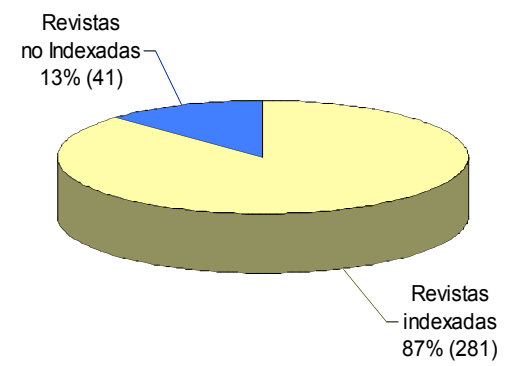

Figura 6. Total de revistas en las que se publica, por tipo de indexación (1997-2006).

De acuerdo con el país de origen de dichas revistas (Figura 7), la producción del Instituto está presente en revistas de 34 países, principalmente, de Estados Unidos (154, el 47.8\%), Reino Unido (84, el 26.1\%), México (81, el $25.2 \%$ ), Holanda (58, el $18 \%$ ), Alemania (16, el $5 \%)$ y España $(13$, el $4 \%)$.

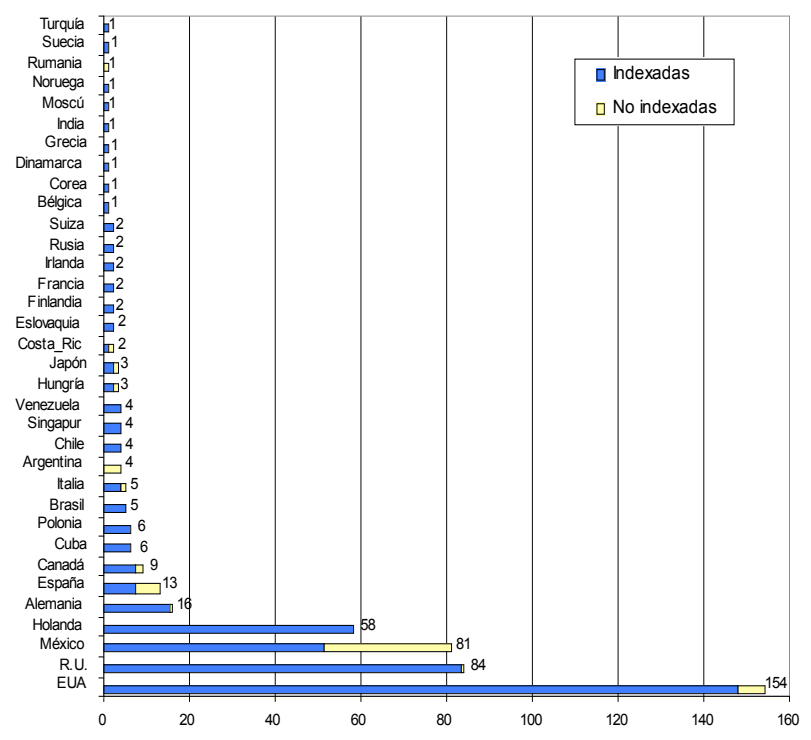

Figura 7. Distribución total de revistas en las que se publica, por país y tipo de indexación (1997-2006) 
Del total de las 322 distintas revistas en las que se ha publicado, se encontró que durante el periodo analizado, 171 de ellas están reportadas con F.I. dentro del ISI y 168 no lo están (Figura 8). Cabe mencionar que las cifras entre ambos grupos contienen duplicidades, debido a que existen casos de revistas que pueden tener F.I. en ciertos años y en otros no.

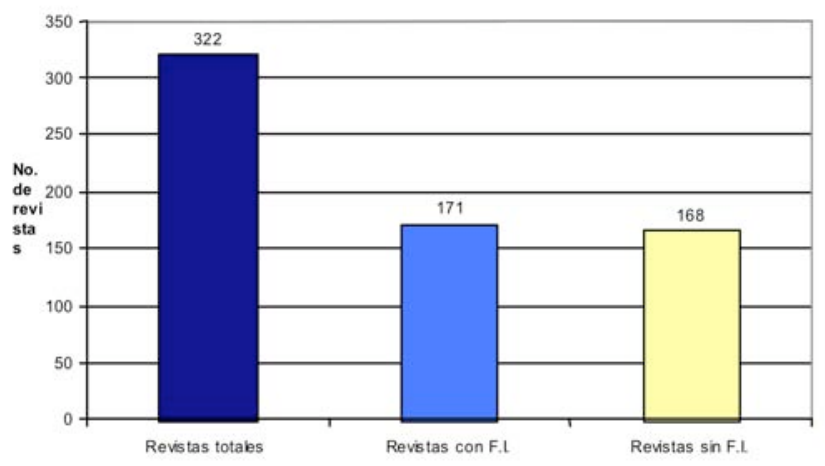

Figura 8. Distribución de revistas respecto al factor de impacto (1997-2006).

El grupo de las 171 revistas con F.I., corresponde a 295 artículos (el $54.7 \%$ del total de artículos). Se identificaron todas las áreas temáticas en las que fueron reportadas por el ISI a lo largo del periodo, haciendo un total de 434 registros temáticos distribuidos en 88 áreas. Posteriormente, se sintetizaron dichas áreas hasta donde se consideró conveniente, resultando una clasificación de 29 áreas y se obtuvieron las frecuencias para cada una de ellas (Figura 9). De esta manera, se encontró que el $56,7 \%$ de las revistas en las que se ha publicado están incluidas en el área de matemáticas, el $38,2 \%$ en el área de física, el $24,6 \%$ en computación, el $22,2 \%$ en ingeniería, el $20,5 \%$ en probabilidad y estadística, el $18,1 \%$ en medicina, el $12,3 \%$ biología, el $9,9 \%$ en mecánica, el $7 \%$ en materiales y en óptica, el $6,4 \%$ en acústica, el 5,8\% en medio ambiente y salud pública, el $2,9 \%$ en genética y herencia, el 2,3\% en química, en ciencias nucleares y tecnología y en geociencias, el $1,8 \%$ en bioquímica y biología molecular, en administración y en señales remotas, el $1,2 \%$ en instrumentación, en sociología, en telecomunicaciones, en termodinámica y en agronomía, y el 0,6\% en antropología, en educación, en imagenología y tecnología fotográfica, en oceanografía y en recursos hidrológicos. lo anterior, hace evidente que la producción científica del IIMAS está incidiendo en diversas disciplinas, tanto científicas como humanísticas.

Como resultado de la construcción del histograma de frecuencias de posiciones $q$ de las revistas donde se ha publicado, que - como se mencionó en la metodología- se formó para representar el número de revistas que está en una posición tal que superan el valor $q$ por ciento de las revistas que integran cierta área temática. En la Figura 10, se observa que de los 434 registros temáticos, se tienen 98 revistas que se encuentran en las primeras posiciones en su área, superando entre el $79,45 \%$ y el $99,31 \%$ (inclusive) de las revistas que integran dicha área. De manera similar, se observa que $109 \mathrm{de}$ las revistas se encuentran en una posición entre el $59,59 \%$ y el $79,45 \%$ (inclusive) de las revistas de su área, 77 de las revistas superan entre el $39,72 \%$ y el $59,59 \%$ (inclusive) de las revistas, 89 de las revistas superan entre el $19,86 \%$ y el $39,72 \%$ y 61 de las revistas están en una posición que supera entre el $19,86 \%$ (inclusive) y la última posición $(0 \%)$.

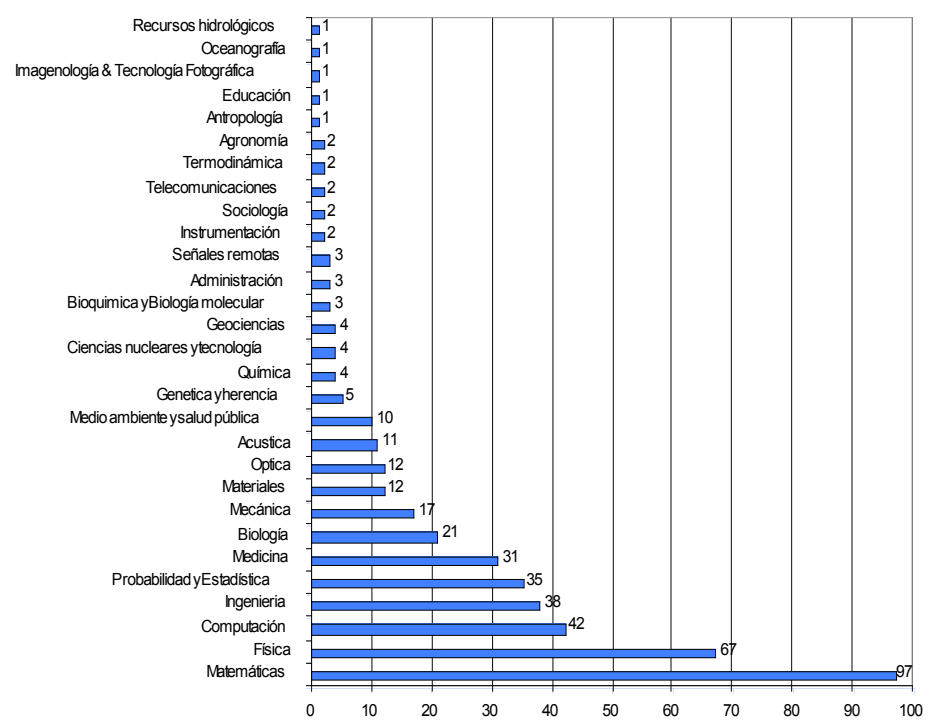

Figura 9. Presencia de la producción del IIMAS según las áreas temáticas de las revistas en las que se publica (1997-2006).

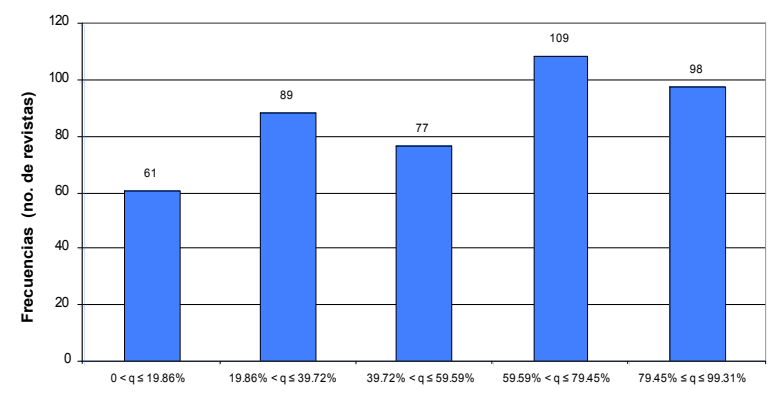

Figura 10. Histograma de frecuencias de posiciones $q$ de las revistas donde se publica (1997-2006). 
Con el objeto de poder visualizar de manera global los F.I. de las revistas en las que se ha publicado, se construyó un histograma de frecuencias del factor de impacto, tomando en cuenta los 295 artículos publicados que cuentan con F.I. La Tabla II muestra las frecuencias de los F.I. de estos artículos agrupados en cinco intervalos, los cuales fueron formados considerado el menor F.I. igual a 0,007 y el mayor igual a 11.295 .

\begin{tabular}{lr}
\hline Intervalos de F.I. & Frecuencia \\
\hline $0.0070<$ F.I. $\leq 2.2646$ & 271 \\
\hline $2.2646<$ F.I. $\leq 4.5222$ & 19 \\
\hline $4.5222<$ F.I. $\leq 6.7798$ & 3 \\
\hline $6.7798<$ F.I. $\leq 9.0374$ & 0 \\
\hline $9.0374<$ F.I. $\leq 11.295$ & 2 \\
\hline
\end{tabular}

Tabla II. Frecuencias del F. I. (1997-2006).

En la Figura 11, se presenta el histograma de frecuencias correspondiente. Donde se observa que el $91,9 \%$ (271) de los artículos que cuentan con F.I. corresponden a revistas que presentan un F.I. en el intervalo $0,0070<$ F.I. $\leq 2,2646$; seguidos del $6.4 \%$ (19) de las que tienen un F.I. en el intervalo $2,2646<$ F.I. $\leq 4,5222$. Y sólo el $1.7 \%$ (5) de ellos corresponden a revistas que tienen un F.I. superior a 4,5222.

Con el fin de poder visualizar de una mejor manera, la forma en que se distribuyen los artículos con F.I. en el intervalo $0.0070<$ F.I. $\leq 2,2646$, se construyó un segundo histograma tomando en cuenta sólo los 271 artículos que lo integran (Figura 12), encontrando que el 62\% (168) de ellos corresponde a revistas con F.I. $\leq 0,910$ y el $46,9 \%$ (127) a revistas con F.I. $\geq 0,910$.

Con respecto a los índices en dónde aparecen las revistas en las que se publica la producción científica del IIMAS, se procedió a identificar los índices donde aparecen cada una de las revistas correspondientes a los 539 registros de artículos para obtener su significancia, obteniendo como resultado un total de 216 índices distintos. En la Figura 13, se presenta la distribución de los 10 índices más significativos, los cuales corresponden a: Current Contents, Cambridge Scientific Abstracts, Compumath Citation Index, Mathematical Reviews, Current Mathematical Publications, Science Citation Index, INSPEC, Applied Mechanics Reviews, Chemical Abstracts y Zentralblatt MATH.

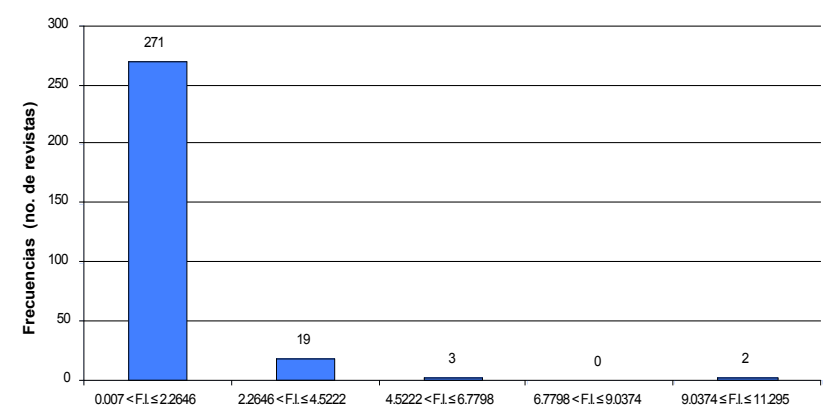

Figura 11. Histograma de frecuencias del F.I. en las revistas donde se publica (1997-2006).

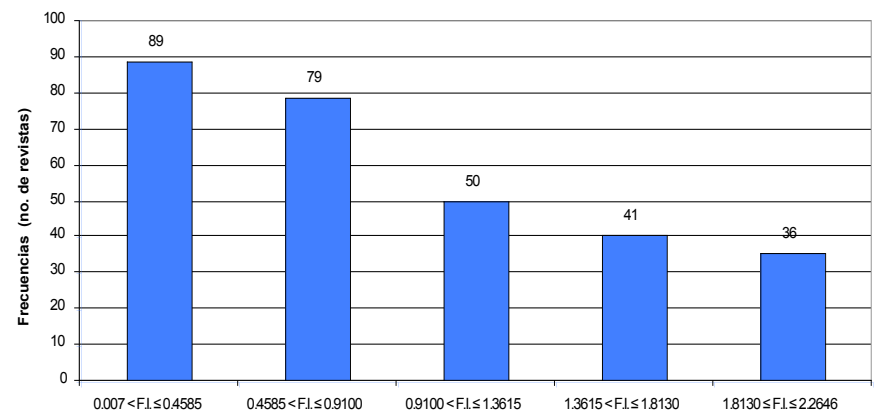

Figura 12. Histograma de frecuencias del intervalo $0.007<$ F.I. $\leq 2,2646$ (1997-2006).

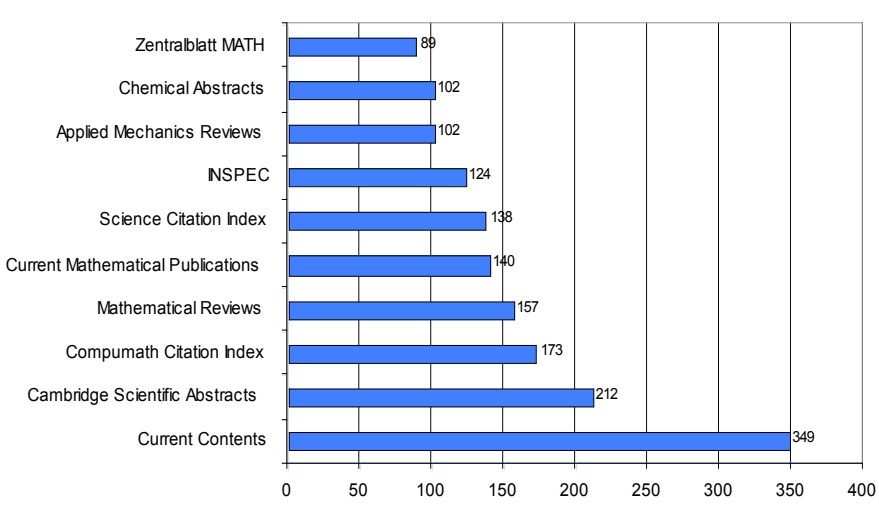

Figura 13. Índices más significativos donde aparecen las revistas en donde se publica (Fuente: Ulrich's, 2007).

\section{Conclusiones}

Del análisis anterior, se puede concluir que la producción científica del IIMAS -conformada por 539 artículos en revistas arbitradas dentro del periodo 1997-2006 - se ha publicado en 322 distintas revistas, originarias de Estados Unidos, Reino Unido, Holanda, México, Alemania y España, principalmente. Las principales áreas en las que se tiene incidencia son matemáticas, física, computación, ingeniería, probabilidad y estadística, medicina, biología y mecá- 
nica, de acuerdo con ISI. Del total de artículos producidos por los investigadores, el $89 \%$ se publica en revistas indexadas y el $54,7 \%$ cuenta con factor de impacto. De estos últimos, el 91,9\% corresponde a artículos en revistas que presentan un F.I. entre 0,007 y 2,2646 , que en su mayoría (el $62 \%$ ) se encuentran por debajo de un F.I. de 0,910.

En cuanto a los índices en los que aparece la producción, se aprecia que se está en los más significativos de la disciplinas que se cultivan en el IIMAS, entre los que destacan INSPEC y Zentralblatt MATH, entre otros.

Finalmente, se observa que la producción global del IIMAS de artículos en revistas arbitradas, durante el periodo analizado, presenta una tendencia creciente.

\section{Referencias}

Avanza la UNAM 21 sitios en la clasificación mundial de universidades; ocupa el lugar 74 del orbe. // Boletín UNAM-DGCS-740 (2006). http://www.dgcs.unam.mx/ boletin/bdboletin/2006_740.html. (2007-05-27)
Informe general del estado de la ciencia y la tecnología. // México: Consejo Nacional de Ciencia y Tecnología, 2006. http://www.siicyt.gob.mx/siicyt/referencias/ muestraEstadisticas.do (2007-05)

Aleixandre-Benavent, Rafael; Valderrama-Zurián, Juan Carlos; González-Alcaide, Gregorio (2007). El factor de impacto de las revistas científicas: limitaciones e indicadores alternativos. // El Profesional de la Información. 16:1 (Enero febrero 2007) 4-11.

Bashan, Yo Av (2002). Publicaciones en revistas de alto impacto internacional. // Ciencia y Desarrollo. 165 (Julio Agosto 2002) 64-73.

Falagas, Matthew E.; Zouglakis, George M.; Papastamataki, Paraskevi A. (2006). Trends in the Impact Factor of Scientific Journals. // Mayo Clinic Proceedings. 81:10 (October 2006) 1401-1402.

Lista de encabezamientos de materia para bibliotecas (1998) Banco de la Republica, Biblioteca Luis Angel Arango. 3a ed. Santafe de Bogotá: R. Eberhard, 1998.

Rodríguez Colmenares, Isabel (2003) Impacto de las revistas científicas en la investigación. // RVG. 8:23 (September 2003) 375-376.

Saint, Sanjay; Christakis, Dimitri (2003). A.Impact factor: a valid measure of journal quality?. // Journal of the Medical Library Association. 91:1 (January 2003) 42.

Seglen, Per O. (2007) Why the impact factor of journals should not be used for evaluating research. // BMJ: British Medical Journal. 314:7079 (2007) 498. 